MS40-P02

\section{A high-throughput crystallisation facility interacting with the new VMXi beamline at the Diamond Light Source}

Halina Mikolajek ${ }^{1}$, James Sandy ${ }^{1}$,Juan Sanchez-Weatherby ${ }^{1}$, Thomas Sorensen ${ }^{1}$

1. Diamond Light Source, Didcot, United Kingdom

email: halina.mikolajek@diamond.ac.uk

A new state of the art crystallisation platform for soluble and membrane proteins has been created within the Research Complex at Harwell combining the expertise of Diamond Light Source, MPL and PPUK. This platform is high-throughput and provides users with an easy route into the VMXi beamline, the new fully automated in situ and serial crystallography beamline at the Diamond Light Source, UK. Users from all over the world have access to this facility and can also deliver their samples for crystallisation experiments. Crystallisation can be set up and stored at $4^{\circ} \mathrm{C}$ as well as at room temperature with humidity control and in lipid cubic phase (LCP) in a number of different plate types. A large number of commercial screens are available and customised screens can be designed and prepared easily. The crystallisation experiments are imaged using Formulatrix Rock Imagers and can be viewed via Rock Maker as well as SynchWeb, which is available on the VMXi beamline. Users can evaluate their crystallisation hits on the VMXi beamline and cycle faster than ever between optimisation cycles to obtain their molecular structure.

Keywords: Crystallisation, Facility, VMXi
MS40-P03

\section{How porous materials improve protein crystallization? Principle of dominant protein-protein adhesion mode}

Jindrich Hasek $^{1}$, Tereza Skálová ${ }^{1}$, Tomáš Kovaí1, Jarmila Dušková ${ }^{1}$, Terézia Kovaĺová ${ }^{1}$, Leona Švecová ${ }^{1}$, Petr Kolenko ${ }^{1}$, Jan Dohnálek ${ }^{1}$

1. Institute of Biotechnology, Czech Academy of Sciences, Praha, Czech Republic

email: hasekjh@seznam.cz

Initiation of crystallization on heterogeneous objects immersed in crystallization drop is well-known, but not well understood phenomenon. In our previous studies [1,2], we have explained details why selection of dominant adhesion mode is generally very important factor in protein crystallization, no matter the crystallization material and the method used. When applied to the protein crystallization in porous materials, it offers a non-conflict explanation of initiation of the crystal-seeds and microcrystals growth in depressions or pores in the surface of heterogeneous materials. The protein molecules adhered specifically at the pore inlets, have restricted access to the adhesion patches oriented toward heterogeneous surface. Uniformity of the remaining adhesion modes exposed into the solution induces also uniformity of the adhesion modes realized in the protein clusters formed at the pore inlet. Thus, the clusters formed in cavities are more regular and stable than those formed in free solution. When the growing crystal-seed reaches its limit size, it breaks off at the pore inlet (the more disordered segment of crystal) and the well-defined microcrystal releases into solution-

The simplest way for preparation of porous material with the required size and shape of cavities is imprinting objects (e.g. proteins) of the required size and shape into the polymer matrix. Theoretically, the cavities 3-20 times larger than the size of a target protein molecule can produce nanocrystal-seeds with sizes in range 50-500 nm. Narrower distribution of cavity sizes is expected to lead to more uniform distribution of crystal sizes. High density of small cavities on the substrate surface seems to be negative, namely when our intention is to grow single crystals. Chemistry of the heterogeneous material decides about the dominant adhesion mode governing the protein crystal growth and responsible for the resulting crystal form.

Experimental observation of the described processes at the structured surfaces or in nano-pores is not possible. However, because our theory and the former interpretations [3] (based on increased protein concentration in cavities) have different impact on design of the "protein-crystal-growth initiators", the prevailing molecular mechanism can be revealed by experiments with well-defined pore sizes and adhesion quality.

The outlined principle of a single dominant adhesion mode and the protein orientation effect in the confined concave space help in a controlled production of protein crystals, e.g. production of a large number of quality microcrystals for serial crystallography at XFEL sources.

Supported by Czech Science Foundation 18-10687S, RVO 86652036 


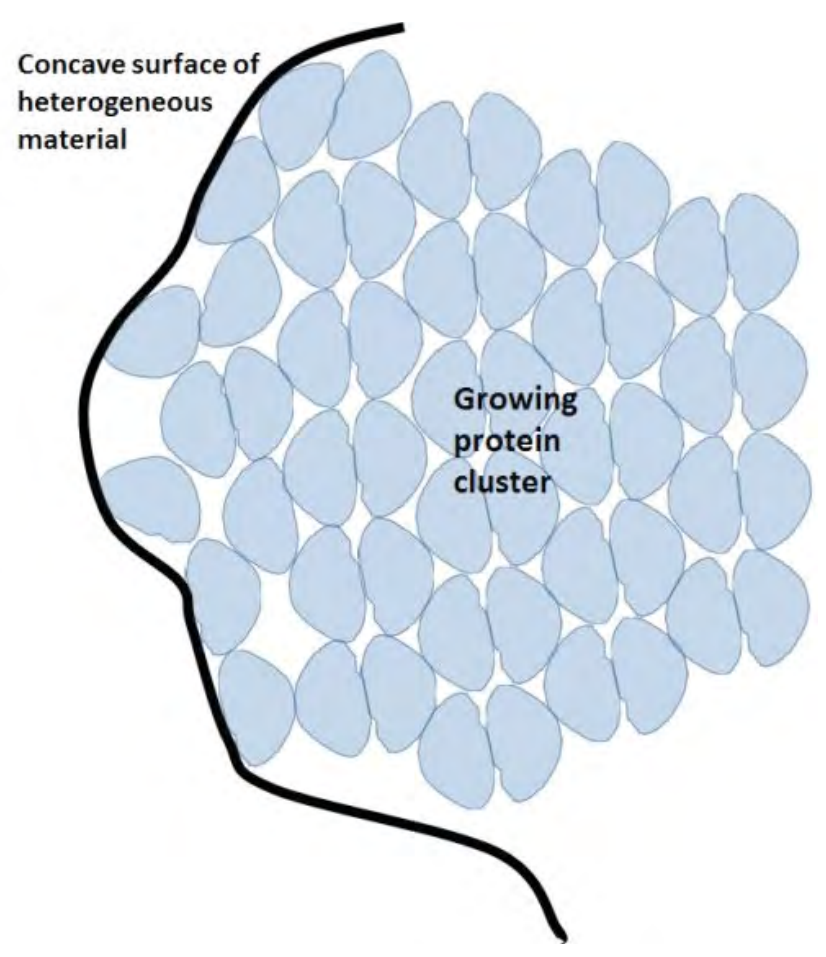

References:

[1] Hašek, J. (2011). J. Synchrotron Radiation, 18, 50-52.

[2] Hašek, J. (2006). Zeitschrift fur Kristallogr. 23, 613-619.

[3] Nanev, C.N. et al (2017). Scientific Reports, 7, 35821-35828.

Keywords: protein crystallization, principle of dominant adhesion mode, porous materials

\section{MS40-P04}

\section{Crystallization of the quadruplex (GGGGTTTTGGGG), with BRACO19}

Rositsa Nikolova ${ }^{1}$, Hristina Sbirkova-Dimitrova ${ }^{1}$, Rusi Rusev ${ }^{1}$, Louiza Dimova $^{1}$, Boris Shivachev ${ }^{1}$

\section{Institute of Mineralogy and Crystallography, Sofia, Bulgaria} email: rosica.pn@clmc.bas.bg

One of the approaches for to development of new anticancer compounds focuses on the design and screening of compounds, small molecules that interact selectively with cancers cells. As telomeres are G-rich noncoding regions located at the end of chromosomes. The telomere shortens with each cell division. When it reaches a critical length it triggers DNA damage response which leads to cell death. Cancer cells have active telomerase (responsible for the maintenance of telomeres) that maintain a shorten but not critical telomere length, provoking those cells to unlimited cell division. G-rich telomeres are in practice forming G-quadruplex structures in vivo. Ligands that selectively bind and stabilize G-quadruplex, are interfering with telomerase activity: maintenance and elongation of telomere. We report on the interaction of the (GGGGTTTTGGGG) sequence with the small molecule BRACO-19. In addition, single crystal of the DNA complex with BRACO19 were grown (Figure 1). The initial crystallization conditions included $\mathrm{KCl}, \mathrm{KCaCO} \mathrm{pH} 7.5, \mathrm{MgCl}_{2}$, Spermine and MPD. The crystals were brownish in color and diffracted up to 2.5 $\AA$ resolution. In order to solve the crystal structure, currently diffraction limit and crystal quality are optimized through modification of the crystallization conditions.

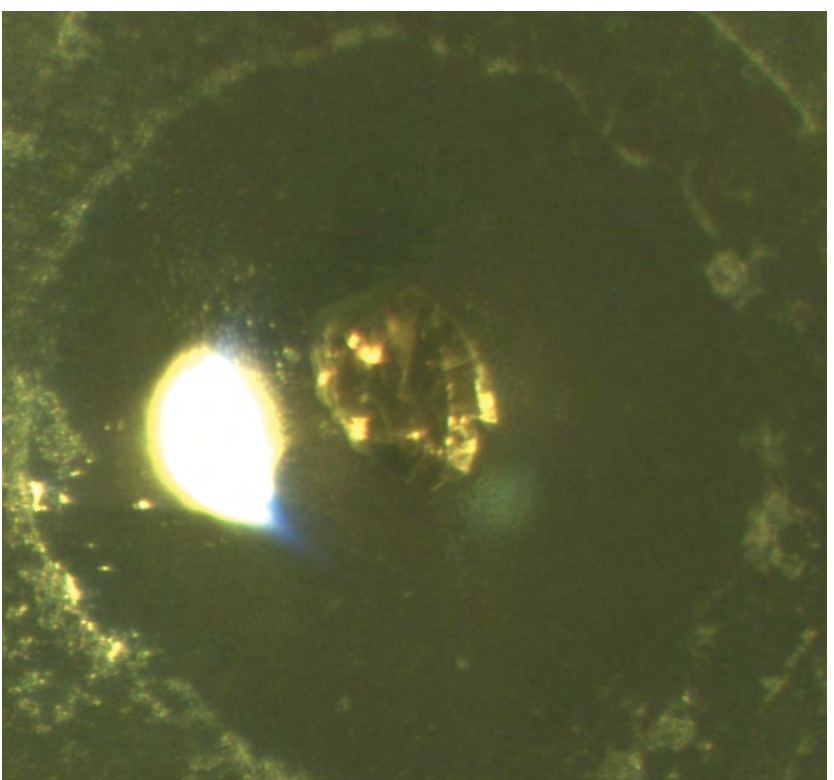

Figure 1. Observed crystals of

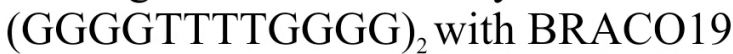

References:

The authors are grateful to the Bulgarian national Science fund grant T02/14 for the financial support.

Keywords: G-4, quadruplex, BRACO19 